\title{
Surrogate Measures to Assess Mobility of People as a Resilience Indicator in Disaster Management: An Exploratory Study in Southeastern Sri Lanka
}

\author{
A. M. Aslam Saja ${ }^{1,3} \cdot$ Melissa Teo $^{1} \cdot$ Ashantha Goonetilleke $^{1} \cdot$ Abdul M. Ziyath $^{1}$. \\ K. W. G. Rekha Nianthi ${ }^{2}$
}

Published online: 18 February 2020

(C) The Author(s) 2020

\begin{abstract}
Understanding social resilience can assist in the formulation of disaster management policies to help communities better prepare for, respond to, and recover from disasters. However, direct social resilience measurement methods such as household surveys are not always a practical option as they are a time- and resource-exhaustive process. Existing measures mainly utilize publicly available census data, which often provide a poor and outdated assessment of current social resilience status. Another limitation includes a failure to capture multiple facets of indicators that are process-oriented and dynamic in nature such as mobility of people. These challenges can be addressed by employing a surrogate approach. Surrogates are alternative measures to depict the target indicator. The surrogate approach can capture key facets of a target indicator, which can be used as potential measures for the target indicator. A framework to conceptualize the surrogate approach is presented, and operationalized using a case study approach on the southeastern coast of Sri Lanka to identify surrogates to measure mobility of people as a resilience indicator. Six higher-order themes were identified as potential surrogates to measure mobility of people in a disaster context. The approach proposed to methodically identify potential surrogates and their measurement protocols can help to improve the current knowledge base
\end{abstract}

Melissa Teo

melissa.teo@qut.edu.au

1 Science and Engineering Faculty, Queensland University of Technology (QUT), Brisbane, QLD 4001, Australia

2 Faculty of Arts, University of Peradeniya, Peradeniya 20400, Sri Lanka

3 Faculty of Engineering, South Eastern University of Sri Lanka, Oluvil 32360, Sri Lanka and understanding of complex interrelationships of social resilience.

Keywords Community resilience $\cdot$ Resilience characteristics - Resilience indicators - Resilience measurements $\cdot$ Social resilience $\cdot$ Sri Lanka

\section{Introduction}

The concept of "resilience" has been increasingly discussed in disaster studies, particularly since the adoption of the Sendai Framework for Disaster Risk Reduction 2015-2030 (UNISDR 2015). The past experiences in preparing for and responding to disasters have necessitated a growing and urgent emphasis on the development of effective methods to measure disaster resilience, so that timely investments in resilience building can be implemented to prevent and mitigate future disaster losses and damages.

Social resilience is defined as the ability of social entities to effectively prepare for, mitigate, and cope with disasters, and to recover from past disasters to reduce the impact of future disaster risks (Bruneau et al. 2003; Rockström 2003; Kwok et al. 2016; Saja et al. 2018). Several frameworks have been proposed during the past two decades to measure social resilience to disasters. However, very few frameworks have been operationalized due to practical difficulties in measuring the proposed resilience characteristics (Beccari 2016). A critical review of 31 social resilience assessment frameworks by Saja et al. (2019) found that only half of the frameworks have been operationalized.

Gregorowski et al. (2017) highlighted three types of challenges in operationalizing resilience measurement 
frameworks: (1) conceptual challenges in translating the complexity of resilience as a concept to practical measurement; (2) technical challenges in selecting and applying methods, or in using special measurement tools, such as data analysis; and (3) practical or logistical challenges in operationalizing a resilience measurement. There is also an increasing state of disconnect, with the practitioner community utilizing more qualitative and participatory approaches to measure resilience, whereas the researchand policy-based resilience frameworks have focused on quantitative measures such as index-based approaches (Gaillard and Jigyasu 2016). The resilience concept is too complex to reduce it to a numeric value, and most of the resilience measures proposed in the research literature are limited to indices and quantitative approaches. While many researchers have proposed a set of prospective indicators and formulae to measure resilience, there is a lack of methodological approach to guide the identification and measurement of those indicators (Bahadur et al. 2010). A robust resilience measure should consider many different aspects of an indicator that includes the availability, functionality, accessibility, quality, and capacity of the resilience phenomenon being measured.

In this context, the "surrogate approach" can be used to identify different facets of an indicator and then select the most important facets (potential surrogates) to address conceptual challenges by translating the abstract and complex concept into a practically applicable measure. The use of surrogates can help to overcome the challenges and limitations in measuring social resilience to disasters (Kulig et al. 2013; Ziyath et al. 2013; Cutter 2016; Sharifi 2016). Developing and translating surrogate measures to real world applications to measure resilience will help in effective planning and resource allocation (Lindenmayer et al. 2015) in disaster management.

A surrogate is defined as an indicator that effectively represents another indicator that is intended to be measured (Rodrigues and Brooks 2007; Miguntanna et al. 2010). The surrogate approach has been successfully used in biodiversity studies, clinical medicine, and environmental studies (Mellin 2011; Barton et al. 2015; Lindenmayer et al. 2015). However, no framework exists in the disaster management literature to explore potential surrogates to assess social resilience. A rigorous methodical use of the surrogate approach has not been investigated yet in the disaster context, which makes this study innovative and explorative. This article presents a surrogate framework to identify important facets of social resilience indicators, and the operationalization of the framework to assess a sample social resilience indicator in southeastern coastal Sri Lanka-mobility of people in the disaster context. The mobility of people in this article is defined as an aspect of movement of people individually or collectively in a disaster situation through available means such as transport facilities (Yamamoto et al. 2018). The potential surrogates explored in this study can capture a relatively comprehensive picture of the target indicator and also offer a uniform approach for understanding and identifying surrogates. The proposed surrogate conceptualization framework has wide applicability in any context to explore potential surrogates to assess resilience.

\section{Social Resilience Assessment Frameworks and Methods}

Many taxonomies of resilience measurements exist that are dependent on the way resilience is defined, framed, and analyzed. Saja et al. (2019) discussed various approaches used to develop resilience frameworks and methods applied in resilience measurement. When the existing methods of measuring resilience are categorized as "topdown" and "bottom-up" methods, for example, the key difference between these two methods is the purpose and application (Schoch-Spana et al. 2019). Top-down methods largely depend on quantitative indicators, which are helpful for resilience investment decision making through comparisons between multiple communities, whereas bottomup methods are driven by a local perspective on resilience and the integration of experiential knowledge that are helpful for the direct local application of results (Cutter and Derakhshan 2019; Schoch-Spana et al. 2019).

Doorn (2017) highlighted that the use of non-aggregated indicators can be more useful for contributing to the detailed analysis of resilience than aggregated indices-the latter are mainly helpful in the evaluation of intervention effectiveness and in drawing attention to an issue. Resilience measurements also need to move beyond existing index-based quantifications to produce results that are oriented towards resilient development outcomes and strategies to enhance resilience at the community level (Peters et al. 2016). New methods are needed for the detailed analysis of resilience indicators that can capture important facets of key social resilience characteristics such as social capital, social mechanisms, and social dynamics (Cutter 2016), all of which are multifaceted, process-oriented, and abstract concepts.

Resilience frameworks that have been operationalized have used direct methods such as household surveys and interviews, and/or data obtained from census reports from public databases to assess resilience. Table 1 highlights key differences of these two widely applied methodsusing direct household surveys and using census measures to assess resilience. The merits in direct household measures are demerits in using census data measures and vice versa. The direct household resilience measures are often 
Table 1 Comparison of methods to assess social resilience

\begin{tabular}{|c|c|c|}
\hline & Resilience measure using household surveys & Resilience measure using census data \\
\hline Data sources & Households & Census data and statistics \\
\hline $\begin{array}{l}\text { Method of } \\
\text { accessing } \\
\text { data }\end{array}$ & $\begin{array}{l}\text { Web/household survey, one-on-one or focus group } \\
\text { interviews, consultation, self-assessment }\end{array}$ & Census reports and data from public databases \\
\hline \multirow[t]{3}{*}{ Merits } & $\begin{array}{l}\text { More realistic at the time of measurement or for a period } \\
\text { of time }\end{array}$ & Minimal use of resources and faster \\
\hline & $\begin{array}{l}\text { Data reflects the perception of social resilience in the } \\
\text { concerned community }\end{array}$ & Easy to develop indices and statistical models \\
\hline & $\begin{array}{l}\text { Can capture process-based measures and qualitative } \\
\text { analysis is possible }\end{array}$ & Reproduced when census is updated \\
\hline \multirow[t]{3}{*}{ Demerits } & $\begin{array}{l}\text { Time-consuming, finance- and human resource-intensive } \\
\text { process }\end{array}$ & $\begin{array}{l}\text { Lack of accuracy compared to surveys, data do not capture key } \\
\text { social dynamics and processes }\end{array}$ \\
\hline & $\begin{array}{l}\text { Replication and regular updates are difficult in resource- } \\
\text { limited contexts }\end{array}$ & $\begin{array}{l}\text { Results can be easily reproduced, replicated, and improved only } \\
\text { when the new census becomes available }\end{array}$ \\
\hline & Limited set of easily measurable indicators are chosen & Mostly captures outcome measures \\
\hline
\end{tabular}

time- and resource-intensive, making them not feasible for regular updates of resilience status (Ziyath et al. 2013). This has resulted in a limited set of easily measurable indicators that often do not comprehensively assess resilience (Saja et al. 2018).

Indirect measures are helpful when it is too complex or not feasible to measure the intended indicator directly (Becker et al. 2015). However, most of the existing social resilience frameworks have used available census data, instead of methodically identifying and selecting key resilience measures that are required in a particular community through a consultative approach with the key stakeholders in disaster management. An analysis by Saja et al. (2019) of social resilience frameworks developed over the last decade, for example, revealed that mobility of people is mostly measured through publicly available census data such as vehicle ownership/percentage of households with a vehicle (Mayunga 2007; Cutter et al. 2010, 2014; Kusumastuti et al. 2014; Burton 2015; Kotzee and Reyers 2016; Qasim et al. 2016), or the existing transportation network and transportation access to the area measured using the length of road per square kilometer of land (Kusumastuti et al. 2014), or the labor force in transportation (such as population employed in special needs transportation services) (Peacock et al. 2010). However, when assessing mobility of people, most of the existing measures fail to provide an explicit focus on transport and evacuation center accessibility for people with special needs, who are the most vulnerable population in any disaster context. Mobility of people in times of disasters is also linked to many other factors, such as early warning, existing social support systems for transport access, and level of awareness of disaster evacuation, all of which are not captured in existing methods, when measuring mobility of people as a resilience characteristic.

The existing census-based proxy measures are not adequate enough to reflect mobility of people in a disaster context, because they do not take into account all key facets of the phenomenon being measured. In many leastdeveloped countries, census data are mostly outdated, sometimes older than a decade, and do not reflect the recent or current status of the community. These limitations constrain the community and disaster management stakeholders in making better-informed decisions before disasters occur, based on a very recent/current status of a community's resilience. In this context, there is a need for a new approach to assess social resilience.

This study conceptualized a surrogate approach to assess a set of selected social resilience indicators as testbeds in a disaster context. In this article, exploring potential surrogates to assess one of the social resilience indicatorsmobility of people-is presented as an example. Potential surrogates can be explored to measure sets of social resilience indicators by consulting key disaster management stakeholders as a one-off exercise. Once the final set of surrogates has been selected, they can be periodically updated in consultation with the relevant agencies at the local level to capture any changes, so that resilience can be robustly measured without repeatedly undertaking resource- and time-intensive processes. 


\section{Conceptualizing the Surrogate Approach for Assessing Mobility of People in a Disaster Context}

Figure 1 shows a framework for conceptualizing a surrogate approach to assess social resilience in disaster management that has been adapted from Lindenmayer et al. (2015) and Birkmann (2006). Two key steps are proposed to guide the logical and systematic identification of surrogates: (1) decision on surrogate approach; and (2) identification of potential surrogates. The decision for a surrogate approach was made because existing methods to assess mobility of people as a resilience characteristic do not capture key facets of mobility of people in a disaster context. This study focused on the identification of key facets of mobility of people that can be used as potential surrogates.

Identification of potential surrogates requires three substeps (Fig. 1): (2.1) Exploring all potential surrogates (answers the "what" question); (2.2) Establishing the surrogacy relationship with the target indicators (answers the "why" question); and (2.3) Defining protocols for measuring surrogates (answers the "how" question). The substeps are explained below.

Substep 2.1 Exploring all potential surrogates: There are three key considerations when exploring all potential surrogates: (1) Select a context. A context needs to be established by: (a) selecting a site (geographical limitation), which could be broadly categorized as urban or rural; and (b) specifying a hazard type or multi-hazard scenario, since resilience measures can vary with different geography or hazard; (2) Select disaster management experts. Experts who are local policymakers and practitioners working in disaster risk reduction and have experience working at the community level should be selected for exploring potential surrogates; and (3) Consultation with experts to collect data to explore potential surrogates.

Substep 2.2 Establishing the surrogacy relationship: This relationship is defined as the extent to which a particular set of features (surrogates) effectively represents a defined set of features of the target indicators (Rodrigues and Brooks 2007). The quality and effectiveness of surrogates depend on the strength of the surrogacy relationship between the target and surrogate indicators (Grayson et al. 1996). The relationship of a potential surrogate with the target indicator is dependent on: (1) The nature of the relationship; whether a surrogate is connected to the target indicator or connected to other surrogates; (2) The strength of the relationship; and (3) How the changes in the surrogacy relationship impact the target indicator. In this substep, the interview participants establish the relationship between the target indicator and potential surrogates. Substep 2.3 Defining protocols for measuring surrogates: The third substep in identifying potential surrogates involves establishing the protocol to guide the measurement to be undertaken. This is based on the consideration of three factors: (1) Type of measurement; whether qualitative, quantitative, or mixed; (2) How to access accurate data, either through primary data collection using sampling or secondary data, or a combination of direct measurement through sampling and secondary data; and (3) Access to reliable sources for surrogate data and how to transform surrogates to measure the target indicator.

\section{Case Study Research}

A qualitative case study method was adopted for this research, because it can help to answer the "how" question to study the complex relationships that underpin social resilience as a dynamic social phenomenon (Marshall and Rossman 2014). The case study research method allowed the exploration of potential surrogates to assess social resilience indicators because it enables delving in-depth into the complexities and processes of how surrogates are perceived, understood, and identified, as well as their potential use in a disaster context (Marshall and Rossman 2014). The use of the surrogate approach is untested in the disaster context, which makes this research exploratory in nature (Boeije 2009). In exploratory research, the consultation of experts who have direct involvement in community-based disaster management work is important, because they can provide practical insights to the problem being investigated.

Based on Creswell (2011), four key aspects of qualitative exploratory research were adopted for selecting the study locations and participants: (1) Setting for the research (case study location); (2) Events - on what the participants are interviewed; (3) Actors-interview participants; and (4) Processes-how the actors are interviewed within the research setting (individual and group).

\subsection{Setting for the Research (Case Study Locations)}

The overall case study region was the southeastern coast of Sri Lanka and four case studies were investigated during September-November 2017. There are three key principles for a data collection strategy in case study research: use of multiple sources of evidence; maintaining a chain of evidence; and creating a case study database (Yin 2013). The selection of the four case studies guided the triangulation of 





Fig. 2 a Case study area in Sri Lanka and the divisional case study locations. b Central/ devolved governance entities in Sri Lanka and their hierarchical linkages

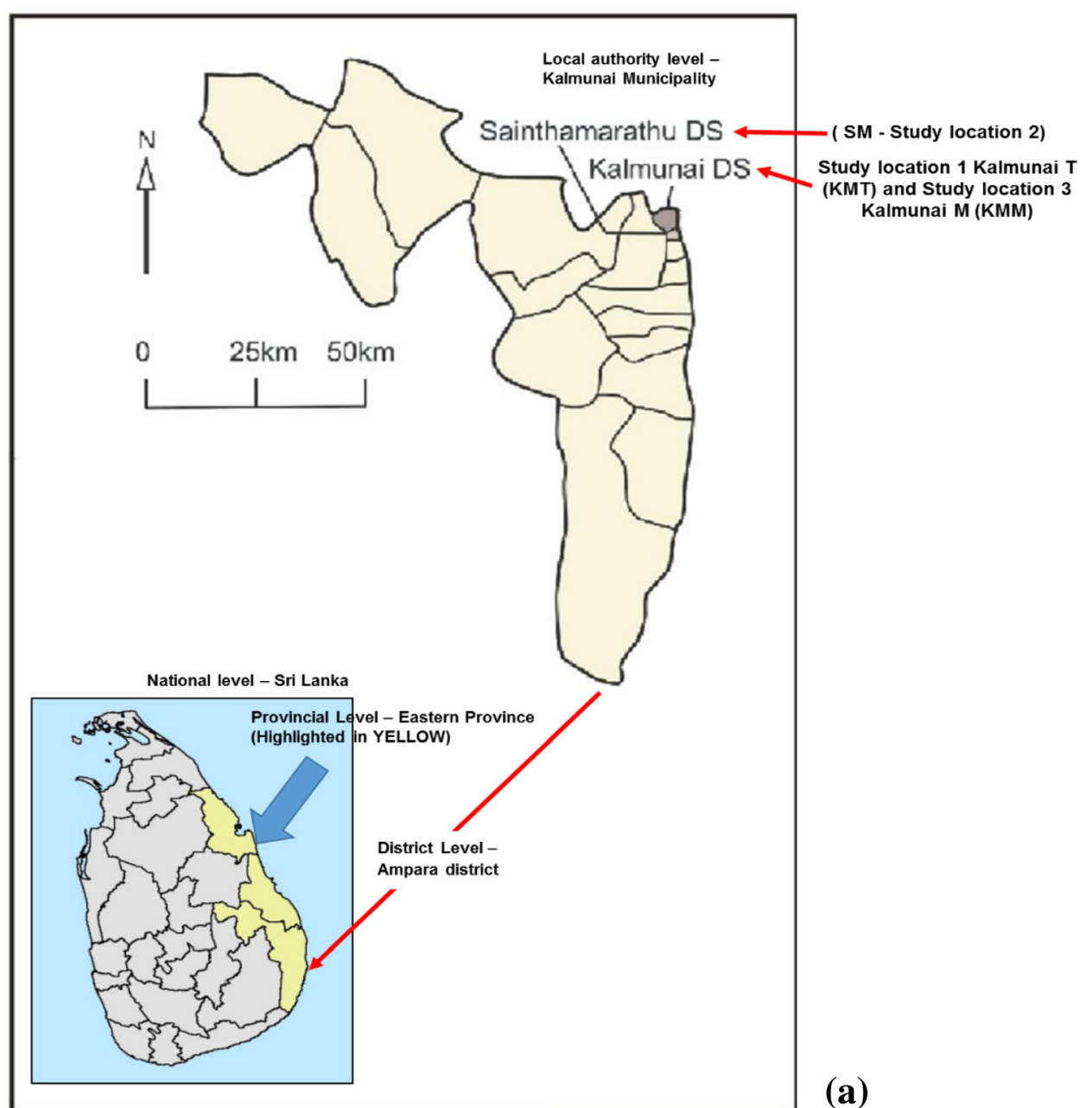

(a)

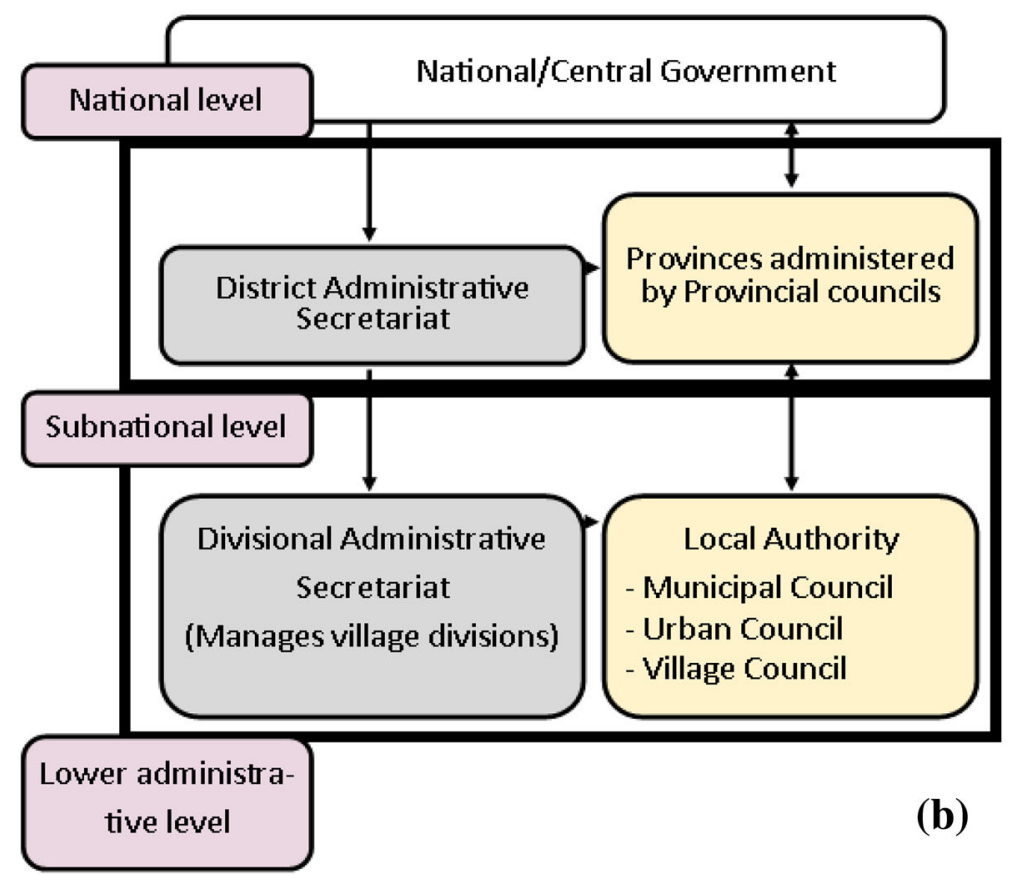


the analysis using multiple cases that reduced biases (Yin 2013) and avoided dominant views among the specific study group.

The global climate risk index 2019 ranks Sri Lanka second among the most climatic disaster-affected countries in the world (Eckstein 2018). Due to rapid urbanization around the world, urban communities are highly exposed to multiple hazards due to a range of factors (Konrad 2003). The three selected case studies at the lower administrative divisional levels are located within Kalmunai Municipality (urban area) in Ampara District in the Eastern Province (Fig. 2a). They were chosen because they are extremely vulnerable and frequently exposed to multiple hazards such as flooding, tsunami, and cyclone (Zubair et al. 2006). The three case study locations are: Kalmunai T (Case study 1, denoted as KMT), Sainthamaruthu (Case study 2, denoted as SM), and Kalmunai M (Case study 3, denoted as KMM) (Fig. 2a).

In addition to the three case study locations at the lower urban administrative levels, a fourth case study focused on the subnational level (includes district and province). This is the next level of governance structure below the national government in Sri Lanka (Fig. 2b). The district level comes under the central government administrative structure, and the provincial level is from the devolved local governance structure in Sri Lanka, and both have roles in disaster management at the subnational level. Figure $2 b$ shows the relationship of the case study locations to the lower, subnational, and national levels in the central administrative structure (in Purple boxes). It also shows the parallel devolved local governance structure at the local authority and provincial levels (in Yellow boxes). The addition of a fourth case study at the subnational level provided deeper insights from the experts working at the higher level of decision making to the overall case study analyses and identified areas for extensive analysis and comparison with the other three case studies at the lower levels.

\subsection{Events (On What the Participants Were Interviewed)}

As the quality of the information is important in case studybased research, interviewing disaster management professionals directly is best suited. Semistructured interviews with open-ended questions were used since these were flexible and likely to yield more new ideas than structured survey-type interviews or less structured interviews (Axinn and Pearce 2006). It is possible to acquire high-quality information in qualitative exploratory research (Johnson and Rowlands 2012) through semistructured interviews because they allow delving into the details of surrogates in a structured way to understand how these are linked to the target indicator and how it can be measured. An interview guide with questions was used to facilitate the interview process and to allow for more probing questions based on the responses. The interview guide included a question structure based on the three substeps in the framework to identify potential surrogates as shown in Fig. 1.

\subsection{Study Actors (Interview Participants)}

Purposive sampling: The knowledge and expertise of disaster management practitioners and policymakers are important in measuring resilience. Purposeful sampling was utilized, and the interviewees were practitioners and policymakers at the local level who had a minimum of 3 years of experience in disaster management work (Creswell 2011). Experts with wide-ranging experience such as working either at the community level or at the subnational policy level and from the government or nongovernmental organizations were sampled, since the richness of the response data can be increased by including diverse stakeholders (Flick 2014).

Sample selection for interviews: The list of members in divisional and district disaster management committees was obtained from the relevant divisional and district government offices. Table $2 \mathrm{a}$, b summarize the participant profiles such as their positions and affiliations. The sample selection also ensured the inclusion of interview participants from different functionalities/departments involved in disaster-related activities, such as social services/protection schemes, disaster relief, village administration, and planning services of a divisional administrative area.

Number of samples: 50 interviews were conducted across the four case studies. A minimum of 10 participants were targeted in each of the case study locations. However, through snow-ball sampling, 10 additional participants were identified as knowledgeable and had vast experience in implementing disaster resilience building projects in the case study locations. In order to strengthen the data, they were also included in the final sample selection (Marshall and Rossman 2014). Table 2c shows the breakdown of interview participants per case study.

\subsection{Interview Process (Groups and Individuals)}

A combination of group and individual interviews were conducted. Study participants were invited for individual interviews when it was difficult to get them into one location to conduct a group interview or when the participant was identified as the person with the most information, who can share a wealth of information if interviewed individually. Interviews were conducted at a location of the experts' choice, which was primarily in their offices. Individual interviews followed the same process and 
Table 2 Interview participant profiles: (a) designation/position, (b) affiliation, (c) breakdown of interview participants per case study in Sri Lanka

\begin{tabular}{|c|c|c|}
\hline (a) Interview participants' designation $(n=50)$ & Number of participants & $\%$ of participants \\
\hline Project director & 18 & $36 \%$ \\
\hline Project manager & 5 & $10 \%$ \\
\hline Project officer & 2 & $4 \%$ \\
\hline President/executive director & 3 & $6 \%$ \\
\hline Project consultant & 5 & $10 \%$ \\
\hline Village administrator & 12 & $24 \%$ \\
\hline Social service officer & 2 & $4 \%$ \\
\hline Divisional disaster relief officer & 3 & $6 \%$ \\
\hline (b) Participants' affiliation $(n=50)$ & Number of participants & $\%$ of participants \\
\hline Central Government & 28 & $56 \%$ \\
\hline Local Government & 2 & $4 \%$ \\
\hline Nongovernmental Organization (Local) & 11 & $22 \%$ \\
\hline Nongovernmental Organization (International) & 7 & $14 \%$ \\
\hline Multilateral agencies such as the United Nations & 2 & $4 \%$ \\
\hline \multicolumn{3}{|l|}{ (c) Interview participants in the case studies $(n=50)$} \\
\hline Case study \# and name & One-on-one interview & Group interview \\
\hline Case Study \#1 & 7 & 1 (3 participants) \\
\hline \multicolumn{3}{|l|}{ Kalmunai T (KMT) } \\
\hline Case Study \#2 & 6 & 1 (4 participants) \\
\hline \multicolumn{3}{|l|}{ Sainthamaruthu (SM) } \\
\hline Case Study \#3 & 6 & 1 (4 participants) \\
\hline \multicolumn{3}{|l|}{ Kalmunai M (KMM) } \\
\hline Case Study \#4 & 20 & N/A \\
\hline \multicolumn{3}{|l|}{ Ampara District/Eastern Province } \\
\hline Total & 39 & 3 (11 participants) \\
\hline
\end{tabular}

semistructured questionnaire structure as the group interviews.

A total of three group interviews and 39 one-on-one interviews were conducted. Each one-on-one interview lasted approximately for 30 to $45 \mathrm{~min}$ and the group interviews took between 45 and $60 \mathrm{~min}$. Group interviews were longer to give each group member a chance to respond to the questions asked. The interview procedure was as follows: questions were posed to the interviewees and the interviewer followed up with additional questions as needed (De Leeuw 2008). All interviews were audiorecorded and conducted in the Tamil language, which is the local language spoken in the case study locations, and because not all participants were fluent in English.

Group interviews: The group interview technique was used with selected government officers who work in the same office (divisional secretariat) or geographical area. It was considered that some participants may gain more confidence in a group setting from the responses of other participants, compared to one-on-one interviews (Axinn and Pearce 2006). Group interviews become efficient when group members are able to stimulate discussion, and can be an effective process to interact with multiple interviewees within a limited time frame (Frey and Fontana 1991). In this research, De Ruyter's (1996, p. 48) definition of group interviews- "a group interview technique which limits respondent interaction to a minimum while attempting to maximize the input of individual group members"-was adopted. The level of interaction between participants is minimal in group interviews, compared to focus group discussions. The interviewer serves as a moderator to encourage each participant to offer new ideas (Axinn and Pearce 2006). A group setting encourages participants to circulate diverse ideas influenced by other group members (Patton 1990). The drawback in group interviews, such as domination by some members in the group, was overcome 


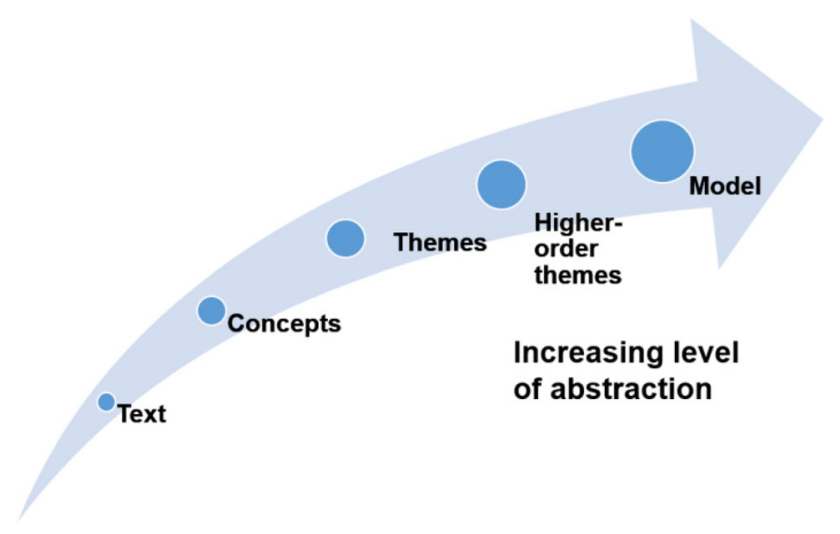

Fig. 3 Levels of interpretation in thematic analysis. Source Adapted from Cruzes et al. (2015)

by providing every member of the group an opportunity to answer every question. In total, 11 government officers (three to four people in each group) participated in group interviews conducted in the three divisional case study locations. The group interviewees were mostly mid-level officers such as village administrators, social service officers, and divisional disaster relief officers from the central government.

Individual interviews (one-on-one/in-person): In order to reach a wide range of geographically and institutionally diverse interview respondents, 39 one-on-one interviews were conducted across the four case studies. During individual interviews, each respondent had more time to explore potential surrogates for the target indicator, compared to the group interviews. Most of the individual interview participants, who were very experienced and knowledgeable in disaster management, provided practical examples from their own experience, compared to the group interview participants.

\subsection{Data Analysis}

The interview audio recordings were transcribed, and then professionally translated from Tamil to English. The translated interview transcripts for each case study were analyzed using the Leximancer data mining tool. Leximancer software ${ }^{1}$ is a computer-based lexical analysis data mining tool, used for content analysis of qualitative data, which can produce thematic strings. Leximancer was used because it provides an automated data analysis based on lexical text properties and is efficient in handling large sets of qualitative data without bias (Sotiriadou et al. 2014). Leximancer is not only more effective than manual coding,

\footnotetext{
${ }^{1} \mathrm{https}: / /$ info.leximancer.com.
}

but also identifies a broad range of concepts, thereby increasing the consistency and reproducibility that helps analysts to derive greater insights (Penn-Edwards 2010; Angus et al. 2013).

The output of the Leximancer analysis is a map that depicts a series of concepts and themes. Concepts in Leximancer are collections of recurrently occurring words that travel together throughout a text document, and a concept map is generated using the frequency of co-occurrences of concepts (Leximancer 2017). A set of concepts that formed a theme was identified from lexi concept maps in a systematic fashion across the entire data set of each case study. The original Leximancer concept/theme map view as it was generated from the transcript data is provided for each case study (Sects. 5.1-5.4). The relevant themes were then interpreted by the researcher using the analyst synopsis window for each theme shown in Leximancer. The analyst synopsis in Leximancer provides the relevant sentences from the transcript data. The themes are heat-mapped, meaning that hot colors (such as red, orange) denote the most important themes, and cool colors (such as blue, green) denote those less important (Leximancer 2017).

The concepts were translated into meaningful themes and a set of correlated themes were then combined to create a higher-order theme. Each higher-order theme was interpreted in terms of potential surrogates that are relevant to the target indicator of measure. The process of thematic synthesis of case study data is shown in Fig. 3 (Cruzes et al. 2015). The levels of interpretation to develop surrogates followed a path of abstraction from text to higherorder themes through interpretation of the concepts and themes generated in the Leximancer analysis as shown in Fig. 3.

The interview transcripts for each case study were collated into a single document for individual analysis. Among many case study analysis techniques, pattern (concepts and themes) matching, explanation building, and cross-case synthesis were used to analyze interview data (Yin 2013). The concepts/themes map using Leximancer was generated for each case study. From these maps, a thematic analysis was carried out by combining the most closely related themes for each case study independently to identify a set of meaningful higher-order themes. A higherorder theme was considered a potential surrogate for each case study area. Each of the higher-order themes built from the distinct case study analysis was explained with interview transcript data.

The interview transcript analysis in Leximancer generated 83 concepts and 41 themes in total across all four case studies. Section 5.1 shows Leximancer concepts/theme maps that were generated from each set of case study data. Each map includes: concepts (shown as nodes and in 
smaller font size), themes (circle around a most prominent single concept/set of concepts and in bigger font size), and higher-order themes (more relevant single theme/combined themes shown within box drawn). For each case study, connected concepts were analyzed with interview transcripts to determine an appropriate theme. In the next stage, interconnected relevant themes were combined to create a higher-order theme.

\subsection{Cross-Case Synthesis and Theory Development}

In order to build a new body of knowledge, a synthesis of multiple cases was necessary. Figure 4, adapted from Yin (2013, p. 60), shows the analysis structure of multiple case studies using cross-case synthesis to develop surrogates based on higher-order themes generated from different case analyses. Unique themes from different case analyses were cross-tabulated to identify commonly occurring higherorder themes across all case studies. Explanation building in multiple case studies was carried out to interpret potential surrogates using surrogacy relationship and protocols for measuring surrogates.

Six unique higher-order themes were identified as potential surrogates from cross-case synthesis. Surrogacy relationship and surrogate measurement protocols for each of the surrogates were explored from the themes and concepts, which are discussed in Sect. 5.2.

\section{Analysis and Discussion: Exploring Potential Surrogates and Surrogacy Relationship from Case Study Analysis}

First, the thematic analysis conducted independently for each case study is discussed in this section. A most relevant quote selected from interview respondents for the respective theme in each case study is provided to describe the relationship between the surrogate and target indicator. Final surrogates identified through cross-case synthesis are then presented at the end of the section.

\subsection{Case Study \#1: Kalmunai T Division (KMT)}

From the thematic analysis of case study \#1, 12 themes were generated and four combined themes (higher-order themes) were identified (Fig. 5) based on connected concept nodes in Leximancer concept maps. They included:

(1) Awareness programs and early warning drills: Many interview respondents highlighted that people who have a higher level of awareness attend evacuation drills annually. For example, Respondent \#23 said that:

Government and organizations have done many awareness programs, many instructions and guides about evacuation and evacuation routes are displayed in streets, and safety evacuation and shelter centers

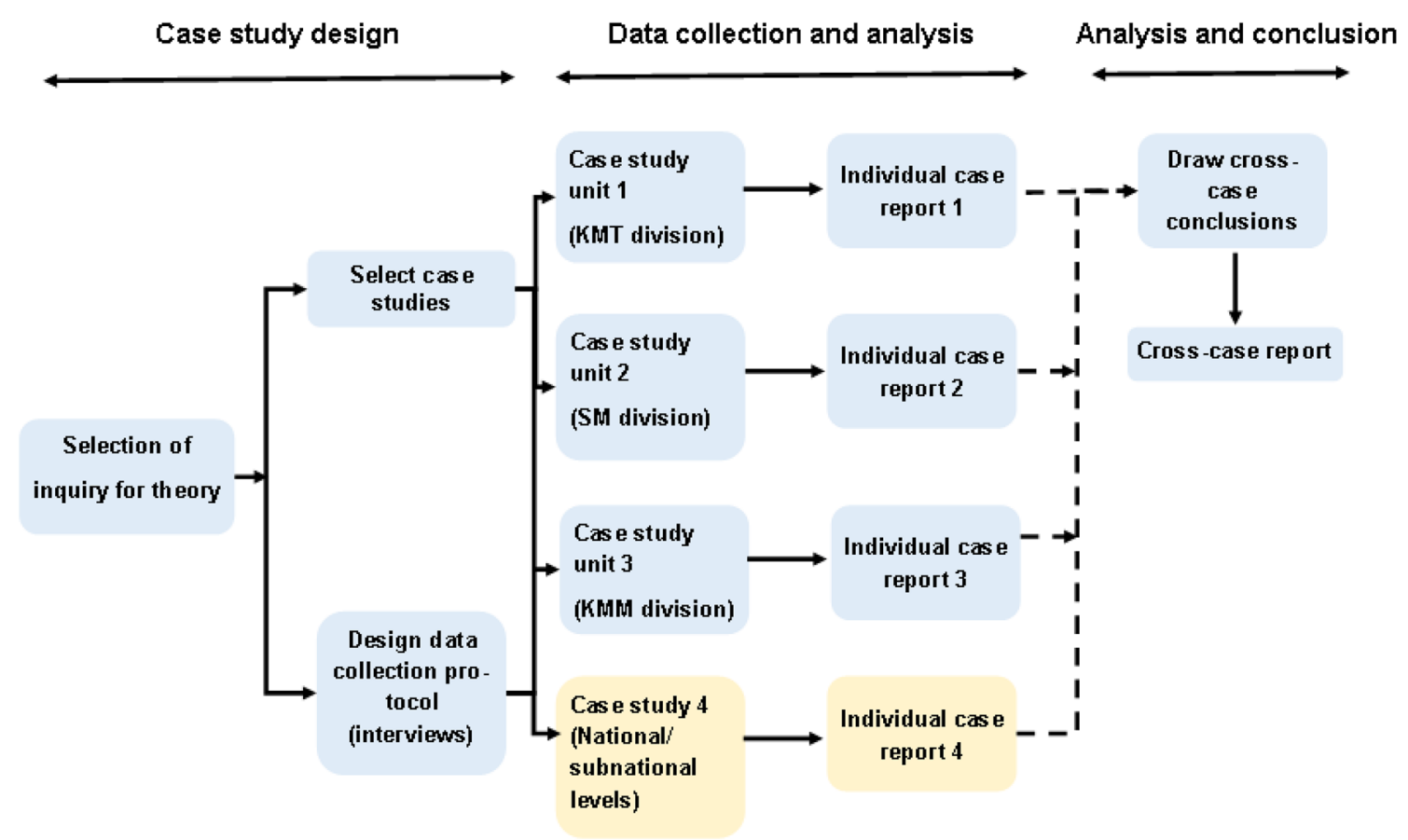

Fig. 4 Case study procedure from design to results in southeastern Sri Lanka (KMT = Kalmunai T; SM = Sainthamaruthu; KMM = Kalmunai M). Source Adapted from Yin (2013, p. 60) 


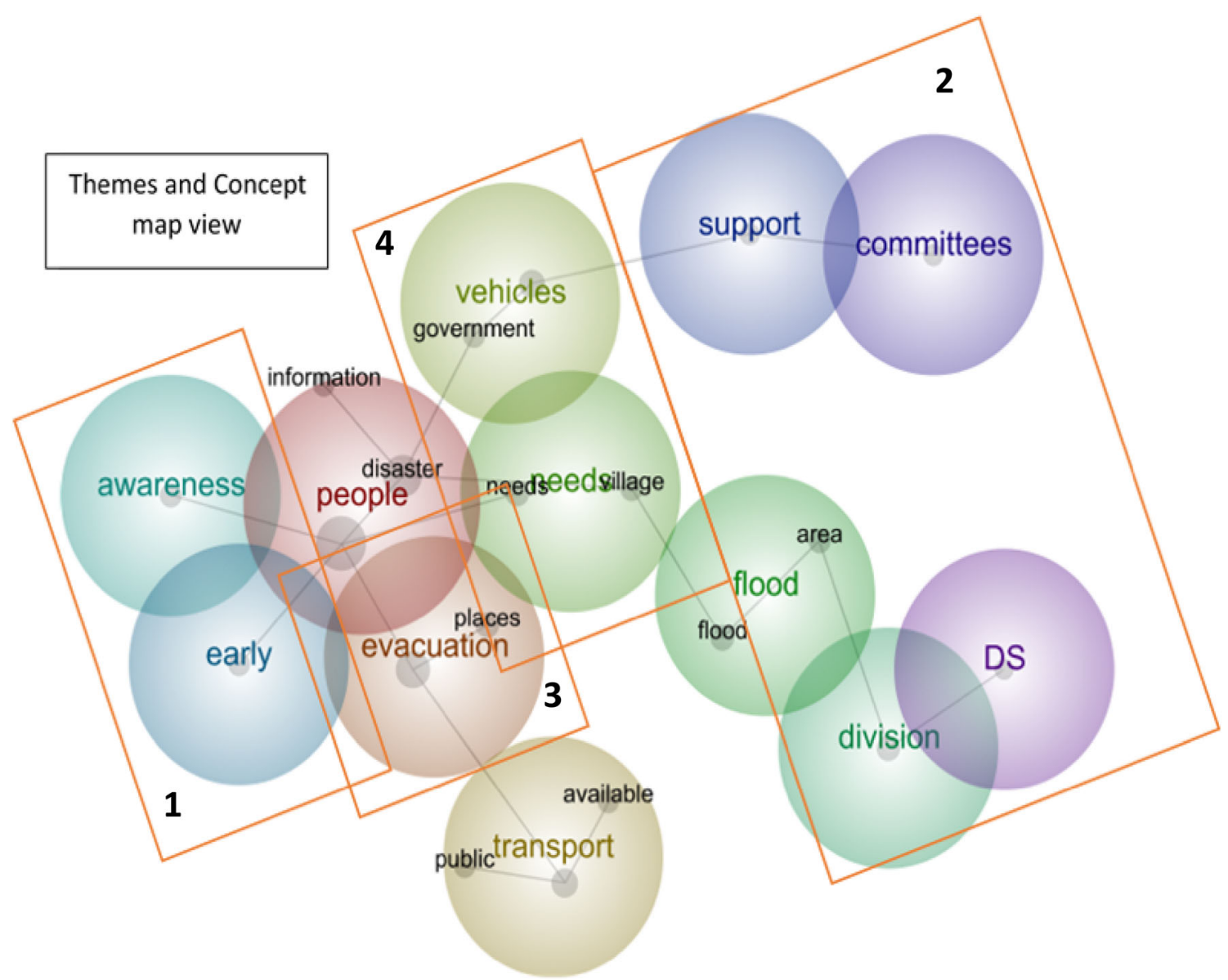

Fig. 5 Original Leximancer concept/theme map view from interview data of case study location \#1, Kalmunai T Division (KMT). DS = Divisional Secretariate. Concepts (shown as nodes and in smaller font size), themes (more relevant single theme/circle around set of concepts and in bigger font size), and higher-order themes (combined themes shown within box) are demarcated and people are made aware and given training, people can now effectively evacuate from their places.

(2) Social support through disaster management committees: This theme mainly refers to the support from the disaster management committees for people to access transport facilities and other mobility assistance during disaster evacuations in the flood affected Divisional Secretariat (DS) divisions. According to Respondent \#27:

From the disaster committees there are arrangements to get access to vehicles. For sick people or emergency cases, accidents, or disasters, we can access vehicles from hospitals and police. Since there is a hospital and military forces within our division, we can get vehicles and there are officers from these government departments who are also disaster committee members.
(3) Evacuation places: This is a stand-alone theme that emerged in this case study. Respondent \#4 highlighted that:

With the participation of the people, we have developed evacuation plans and maps to identify safer places.

(4) Transport for people with special needs: Vehicles targeting people with special needs are two interrelated themes in this case study, as emphasized by Respondent \#34:

Disaster management committees in each division can organize some transport facilities for people with special needs such as elders, children, and the disabled based on the transport services and facilities available within the area. We cannot provide transport facilities for all people and we have to give priority. 


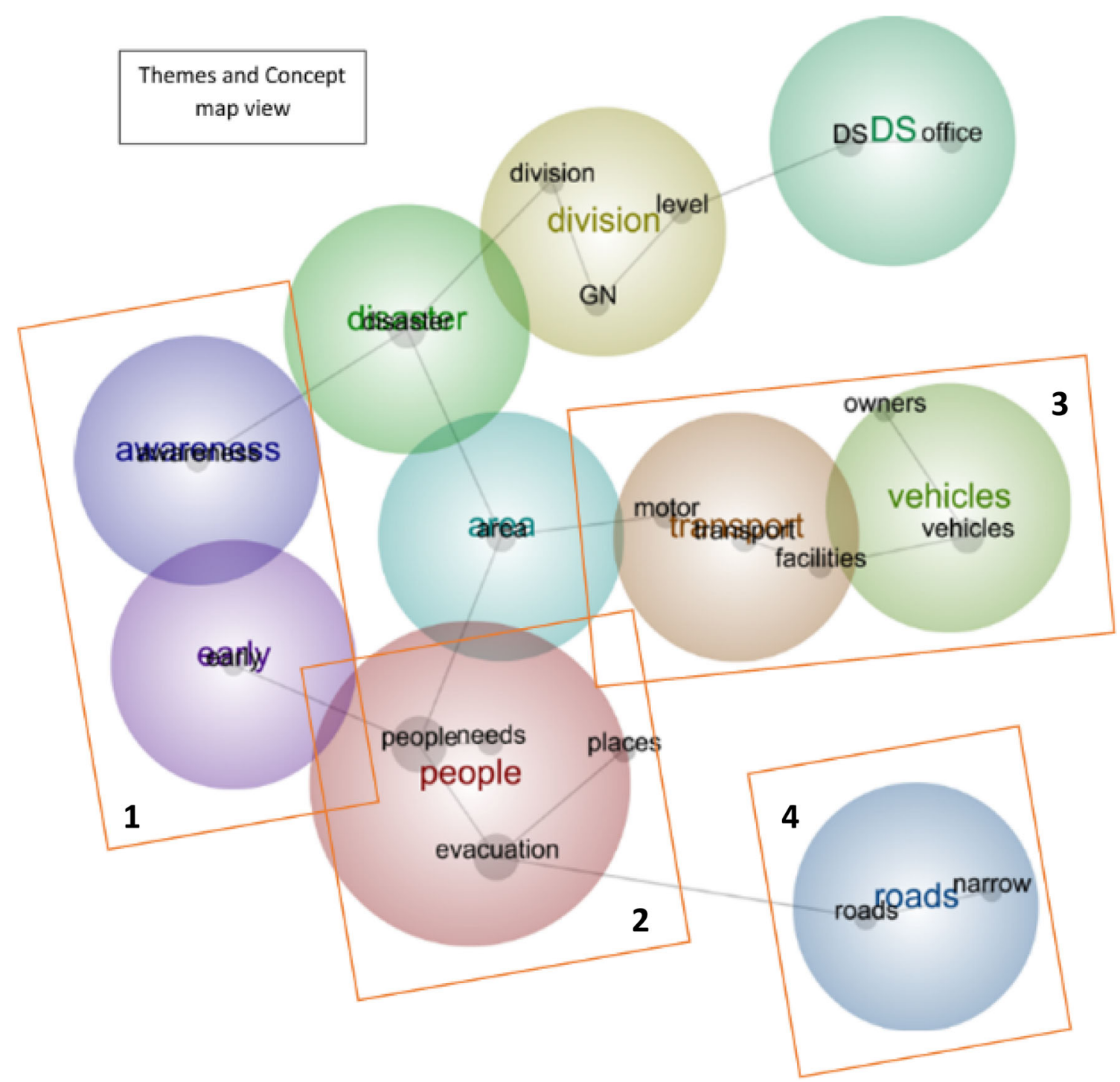

Fig. 6 Original Leximancer concept/theme map view from interview data of case study location \#2, Sainthamaruthu Division (SM). DS $=$ Divisional Secretariate. Concepts (shown as nodes and in smaller font size), themes (circle around set of concepts and in bigger font size), and higher-order themes (more relevant single theme/combined themes shown within box)

\subsection{Case Study \#2: Sainthamaruthu Division (SM)}

Ten distinct themes were generated from case study \#2. By combining closely related themes, four higher-order themes were identified (Fig. 6). The higher-order themes included:

(1) Awareness and early warning: Similar to theme (1) in case study \#1, this theme was also generated in this case study. Respondent \#6 highlighted that:

Evacuation routes and maps have been displayed in many villages. This awareness has been raised through programs as to how to evacuate and where to evacuate. This was done using pictures and they were also given instructions.

(2) Evacuation places with facilities for people with special needs: This theme emphasized the importance of evacuation places with facilities for people with special needs. According to Respondent \#12:

We have an evacuation center now in our division. It is now being constructed with facilities for special needs... In the past we used schools and mosques as evacuation centers. But now we can use this new evacuation center built for the purpose of accommodating people when disasters occur. 


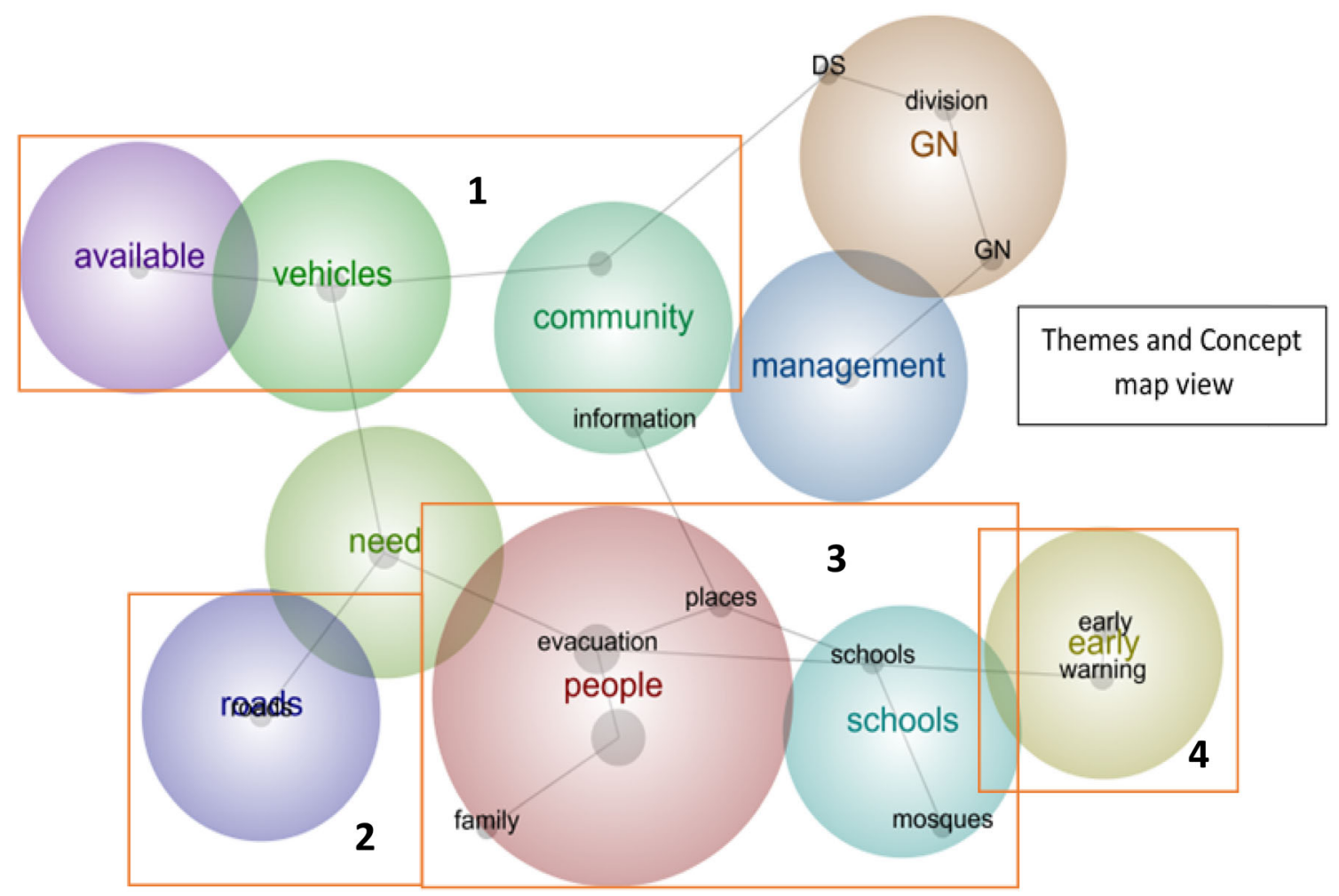

Fig. 7 Original Leximancer concept/theme map view from interview data of case study location \#3, Kalmunai M Division (KMM). DS = Divisional Secretariate; GN = Grama Niladhari division. Concepts (shown as nodes and in smaller font size), themes (circle around

(3) Transport facilities and vehicle owners: Vehicle ownership and owner willingness to support during disasters emerged as interrelated themes in this case study. Respondent \#6 emphasized that:

Van and bus owners are there in my area, a bus owner with five buses has given his buses for evacuation services free of charge. A voluntary contribution. Similarly, tractor owners also have done the same. If we can get the details of vehicle owners within the community, that will help to mobilize help in transport during disasters.

(4) Narrow roads: The theme of community infrastructure that is important for mobility of people, such as road networks and bridges connecting roads emerged in this case study. Respondent \#15 highlighted this:

There are problems to use vehicles in massive scale due to narrow roads... For example, if a person has a van, when they evacuate they will take some more people with them. For cars and motorbikes, they have to evacuate alone. Many roads are narrow, and our bridges are very narrow, so that is not enough in a large evacuation. set of concepts and in bigger font size), and higher-order themes (more relevant single theme/combined themes shown within box)

\subsection{Case Study \#3: Kalmunai M Division (KMM)}

Leximancer analysis generated 10 themes for case study \#3 and four combined themes were found useful as surrogates (Fig. 7). The four higher-order themes found in case study \#3 include:

(1) Vehicles available in the community: This theme highlights the existing vehicle capacity in the division that can be utilized during disaster evacuation. According to the opinion of Respondent \#9:

We should check whether there is a local level mapping for availability of vehicles and resources at the community level. Where the vehicles are available and how many are available in each village. We have to check if the available vehicles have the ability to evacuate people with special needs.

(2) Evacuation roads: This theme is related to evacuation routes and planning. Respondent \#25 explained that:

We have to first talk about roads for effective evacuation. When people evacuate, if there are any problems from the private lands that create problems 


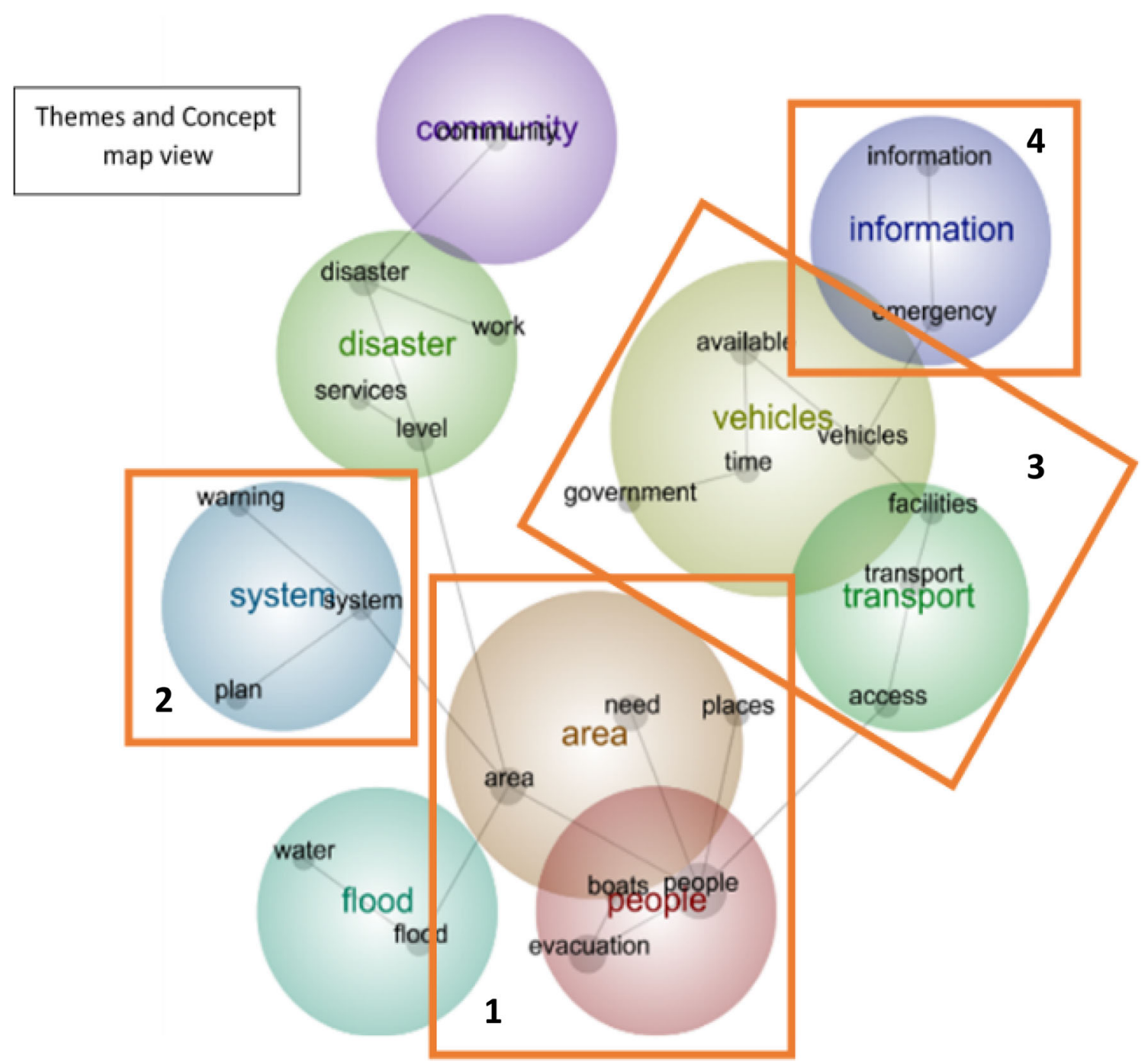

Fig. 8 Original Leximancer concept/theme map view from interview data of case study location \#4, Ampara District/Eastern Province (Subnational Level), Sri Lanka. Concepts (shown as nodes and in smaller font size), themes (circle around set of concepts and in bigger font size), and higher-order themes (more relevant single theme/combined themes shown within box) to roads, we provide compensation to those private owners and get the land for roads to make the road network effective and smooth during disasters.

(3) Evacuation to mosques and schools: These themes are related to using public buildings such as places of worship and schools in times of disasters. According to Respondent \#29:

... People went to mosques and schools when there was a flood. The household heads stayed back and removed belongings and safeguarded their belongings from floodwater. People with special needs were taken by the family members to mosques and schools.
(4) Early warning: Early warning was emphasized by many interview participants in case study \#3 as an important facet in determining mobility of people and access to transport. Respondent \#32 said that:

Early warning towers are built and equipment for early warning signs is given. The towers are regularly tested and rehearsal programs are being held frequently. People are trained and awareness raising programs are also held. Evacuation boards are displayed as to how people should be evacuated. 
Table 3 Surrogates (higher-order themes) mapping against individual case studies

\begin{tabular}{|c|c|c|c|c|c|c|}
\hline \multirow[t]{2}{*}{ Case study locations/area } & \multicolumn{6}{|c|}{$\begin{array}{l}\text { Surrogates to measure mobility of people (higher-order themes) } \\
\text { (The names of surrogates S1 to S6 are provided below the table) }\end{array}$} \\
\hline & $S 1$ & $S 2$ & $S 3$ & $S 4$ & S5 & S6 \\
\hline Case study 1 & $\mathrm{X}$ & $X$ & $\mathrm{X}$ & & $X$ & \\
\hline \multicolumn{7}{|l|}{ Kalmunai T (KMT) } \\
\hline Case study 2 & $\mathrm{X}$ & $\mathrm{X}$ & $\mathrm{X}$ & $\mathrm{X}$ & & \\
\hline \multicolumn{7}{|l|}{ Sainthamaruthu (SM) } \\
\hline Case study 3 & $\mathrm{X}$ & $X$ & $\mathrm{X}$ & $\mathrm{X}$ & & \\
\hline \multicolumn{7}{|l|}{ Kalmunai M (KMM) } \\
\hline Case study 4 & $\mathrm{X}$ & $X$ & $\mathrm{X}$ & & & $X$ \\
\hline \multicolumn{7}{|l|}{ Ampara District } \\
\hline Eastern Province (subnational level) & & & & & & \\
\hline
\end{tabular}

S1: Available transport facilities (targeting people with special needs)

S2: Evacuation places and centers (including facilities for people with special needs)

S3: Awareness programs/plans and early warning systems

S4: Evacuation routes and plans

S5: Social support systems (including from disaster management committees and government)

S6: Emergency information dissemination and sources (including emergency telephone numbers of transport facilities)

\subsection{Case Study \#4: Ampara District/Eastern Province (Subnational Level)}

For case study \#4, nine themes were generated and four combined higher-order themes were found useful as surrogates (Fig. 8). These included:

(1) Evacuation places in the area: This theme highlights the importance of designated safe areas as part of a disaster preparedness plan to increase the resilience of communities. Respondent \#17 emphasized:

People go to nearby common places like a temple, school, community hall, and temporary center. There are places that are identified by the Disaster Management Center and village disaster management committees (VDMC) for people to evacuate in times of disasters.

(2) Early warning systems and plans: The theme on available early warning mechanisms and proper execution plans when there is a disaster emerged in case study \#4, which was pointed out by many interview respondents. Respondent \#5 stated that:

Early warning group members' details should be available at the DS (Divisional Secretariate) and village level which is an important indicator. The availability of contact directory of early warning group in GN (Grama Niladhari division) divisions and being given identity cards is another indicator. There is also a software system with contact details of key people in the early warning group at the division level.

(3) Availability and access to transport facilities: One of the key factors to understanding mobility of people is to know the availability and access to transport facilities in times of disasters. Respondent \#5 highlighted this:

We do resource mapping for a village. We also collect information such as how many vehicles and where they are in the households, how many tractors, how many carts are available and where they are when we do village development plans, and include this information in emergency preparedness plans.

(4) Emergency information sources: Access to information is key to improving the mobility and access to required transport facilities during disaster warnings. According to Respondent \#13:

Nowadays the information system is dependent on mobile phones... Disaster Management Committee will have information on how to access transport or bus. Disaster Management Center has some information district-wise and people can call DMC and ask them for help to access transport. 


\subsection{Final Surrogates Identified through Cross-Case Synthesis}

Table 3 summarizes the mapping of higher-order themes (taken as surrogates) that emerged from the thematic analysis of the interview data of the four case studies.

For each of the potential surrogates, a brief description and assessment protocols from the synthesis of the interview responses are summarized below.

Available transport facilities (targeting people with special needs) (S1): This surrogate was highlighted in all four case studies. Access to a transport facilities should be planned to deal with future disasters effectively. Timely access to a required transport facility is necessary for effective evacuation and mobility during and after disasters. The availability of transport facilities or timely access to transport facilities for people with special needs (PwSN) was highlighted by many interview participants in the case studies. The measurement of the surrogate $(S 1)$ should include the assessment and mapping of the ratio of vehicle ownership to population, availability of vehicles to "transport PwSN" to the "PwSN" ratio, and the ratio of vehicles available with government institutions and private owners that can be used during disasters.

Evacuation places and centers (including facilities for people with special needs) (S2): This surrogate was highlighted in all four case studies. The existence of demarcated evacuation places or purpose-built disaster evacuation centers can help the population at risk to execute a planned evacuation during disasters. The level of awareness among the people about the facilities at the evacuation centers will influence the mobility of people with special needs who are the least resilient segment of the population. The surrogate (S2) measurement protocol can include: assessing and mapping of evacuation places available (places of worship/schools/public buildings/designated buildings) and their capacity, ratio of evacuation places to how many are equipped to handle people with special needs, and the ratio between the total capacity of evacuation places and the population at risk.

Awareness programs/plans and early warning system (S3): This surrogate was also highlighted in all four case studies. People at-risk should be made aware about the mobility of and accessibility to transport facilities when required during a disaster warning or response. Awareness programs can influence the way people evacuate and the resilience of the community to disasters by planning for effective evacuation during disasters. The dissemination of early warning messages at the right time to the population at risk can help people to evacuate to safer places. Effective risk communication will result in greater mobility and allow people to prepare for timely evacuation. The surrogate (S3) can be measured by mapping and assessing the existence of early warning mechanisms such as towers, sirens, and public address systems in places of worship and schools; annual disaster evacuation drills (ratio of people who participated to the population at risk, number of people evacuated carrying belongings); number of training and awareness programs related to evacuation conducted annually in places of worship and schools; and participation trends in training and awareness programs conducted related to evacuation.

Evacuation routes and plans (S4): This surrogate was highlighted in case studies \#2 and \#3. The existence of evacuation routes and plans help a community to be resilient when disasters occur, so that the community can respond to the event effectively. The evacuation plan is often part of the bigger disaster management plan and identifies the requirement for transport facilities for evacuating people in the event of a disaster. The understanding of evacuation access routes and multiple evacuation access points will contribute to greater mobility of the community as a whole, as highlighted by many interview participants across the four case studies. The surrogate $(S 4)$ can be measured by mapping the key access roads including multiple access points that are connecting to major highways, and assessing the road conditions, road infrastructure, and evacuation signage with instructions. Village or street disaster management committees and existence of evacuation plans available with these committees can also be assessed.

Social support systems (including from disaster management committees and government) (S5): The social support system (support through disaster management committees and government officials) was selected as the fifth surrogate from case study \#1. The support for accessing transport and assisting in evacuation after a disaster warning from the neighboring community and other support systems such as police and security forces is another important factor for greater mobility. The support for accessing transport and assisting in the mobility of people to evacuate from emerging disasters may also come from within the community. People who are not at risk from the emerging disaster, such as people living in less vulnerable areas within the same community, may come forward to assist people who are living in highly vulnerable areas. The surrogate (S5) can be measured by assessing the resource maps available in the village-based disaster management plans, the existence of annually elected village level disaster management committees, and the activities of disaster management committees, helping to increase the mobility of people for disaster evacuation.

Emergency information dissemination and sources (including emergency telephone numbers of transport facilities) (S6): Emergency information sources were selected as the sixth surrogate to measure access to a transport facility 
from case study \#4. The dissemination of information to people at risk is an important factor for timely access to a transport facility for evacuation during a disaster early warning. Access to information such as emergency telephone numbers of the key organizations involved in disaster response and recovery is needed to access required resources such as emergency transport facilities for evacuation. The surrogate (S6) can be measured by sampling the area-based social media platforms/websites/groups, and assessing the active disaster information dissemination services and programs, the number of people who use the internet or other emergency communication devices such as satellite phones and their usage during the disaster warning period, and the usage of area-based emergency call number services for disaster information and requests for emergency transport facilities.

\section{Conclusion}

A framework to conceptualize the surrogate approach used in this research provides a novel method to measure social resilience indicators. The surrogate conceptualization framework was operationalized to measure mobility of people in times of disasters using a case study on the southeastern coast of Sri Lanka. The surrogate conceptualization framework includes two key steps: (1) decision for surrogate approach; and (2) the identification of potential surrogates, which can provide a fairly adequate measure of a complex and abstract resilience indicator.

The study initially identified 83 concepts and 41 themes from four case studies. Similar themes were then aggregated and six higher-order themes were identified through cross-case synthesis that can be considered as potential surrogates to measure mobility of people. Three surrogates - transport facilities available (targeting people with special needs), evacuation places and centers, and awareness programs and early warning systems-have high validity, since they were identified across all four case studies. Another set of relevant higher-order themes (evacuation routes and plans, social support systems, and emergency information sources) were found in at least one of the case studies, which can also be potential surrogates to measure mobility of people in a disaster context. Six potential surrogates to measure mobility of people are highly reliable and practically applicable in similar contexts, and they were identified in consultation with practitioners and policymakers who are highly experienced in implementing disaster management activities at the community level.

The proposed surrogate conceptualization framework and the process of operationalizing it provides a uniform approach among different stakeholders and has wide applicability in any context to explore potential surrogates to assess resilience. It will also assist practitioners to further advance social resilience measurement to devise effective resilience building plans, and make appropriate resilience building resource allocations. The potential surrogates that emerged in this study can have a broader use in similar contexts and in different geographical or hazard scenarios through an appropriate contextualization process. The potential surrogates identified in this study can be applied in different contexts to improve the assessment of mobility of people in disaster management. Future research also needs to test the surrogate approach to other social resilience indicators to advance the resilience assessment knowledge in disaster management.

Acknowledgements We acknowledge the University Grants Commission of Sri Lanka (UGCSL) and Queensland University of Technology (QUT), Australia for providing a research scholarship to the first author for undertaking this study.

Open Access This article is licensed under a Creative Commons Attribution 4.0 International License, which permits use, sharing, adaptation, distribution and reproduction in any medium or format, as long as you give appropriate credit to the original author(s) and the source, provide a link to the Creative Commons licence, and indicate if changes were made. The images or other third party material in this article are included in the article's Creative Commons licence, unless indicated otherwise in a credit line to the material. If material is not included in the article's Creative Commons licence and your intended use is not permitted by statutory regulation or exceeds the permitted use, you will need to obtain permission directly from the copyright holder. To view a copy of this licence, visit http://creativecommons. org/licenses/by/4.0/.

\section{References}

Angus, D., S. Rintel, and J. Wiles. 2013. Making sense of big text: A visual-first approach for analysing text data using Leximancer and Discursis. International Journal of Social Research Methodology 16(3): 261-267.

Axinn, W.G., and L.D. Pearce. 2006. Mixed method data collection strategies. Cambridge: Cambridge University Press.

Bahadur, A.V., M. Ibrahim, and T. Tanner. 2010. The resilience renaissance? Unpacking of resilience for tackling climate change and disasters. SCR discussion paper 1. https://assets.publishing. service.gov.uk/media/57a08b1ce5274a27b2000973/SCR-Discus sionPaper1-resilience-renaissance.pdf. Accessed 16 Jan 2020.

Barton, P.S., J.C. Pierson, M.J. Westgate, P.W. Lane, and D.B. Lindenmayer. 2015. Learning from clinical medicine to improve the use of surrogates in ecology. Oikos 124(4): 391-398.

Beccari, B. 2016. A comparative analysis of disaster risk, vulnerability and resilience composite indicators. PLoS Currents. https://doi.org/10.1371/currents.dis.19f9c194f3e3724d9ffa285b1 57c6ee3.

Becker, D., S. Schneiderbauer, J.M. Forrester, and L. Pedoth. 2015. Guidelines for development of indicators, indicator systems and provide challenges. Louvain, Belgium: CRED (Centre for Research on the Epidemiology of Disasters). 
Birkmann, J. 2006. Measuring vulnerability to natural hazards: Towards disaster resilient societies. Tokyo: United Nations University Press.

Boeije, H. 2009. Analysis in qualitative research. London: Sage Publications.

Bruneau, M., S.E. Chang, R.T. Eguchi, G.C. Lee, T.D. O'Rourke, A.M. Reinhorn, M. Shinozuka, K. Tierney, W.A. Wallace, and D. von Winterfeldt. 2003. A framework to quantitatively assess and enhance the seismic resilience of communities. Earthquake Spectra 19(4): 733-752.

Burton, C.G. 2015. A validation of metrics for community resilience to natural hazards and disasters using the recovery from Hurricane Katrina as a case study. Annals of the Association of American Geographers 105(1): 67-86.

Creswell, J.W. 2011. Research design: Qualitative, quantitative, and mixed methods approaches, 3rd edn. London: Sage Publications.

Cruzes, D.S., T. Dybå, P. Runeson, and M. Höst. 2015. Case studies synthesis: A thematic, cross-case, and narrative synthesis worked example. Empirical Software Engineering 20(6): 1634-1665.

Cutter, S.L. 2016. The landscape of disaster resilience indicators in the USA. Natural Hazards 80(2): 741-758.

Cutter S.L., and S. Derakhshan. 2019. Implementing disaster policy: Exploring scale and measurement schemes for disaster resilience. Journal of Homeland Security and Emergency Management 16(3): Article 20180029.

Cutter, S.L., K.D. Ash, and C.T. Emrich. 2014. The geographies of community disaster resilience. Global Environmental Change 29: $65-77$.

Cutter, S.L., C.G. Burton, and C.T. Emrich. 2010. Disaster resilience indicators for benchmarking baseline conditions. Journal of Homeland Security and Emergency Management 7(1): Article 51.

De Leeuw, E. 2008. The SAGE handbook of social research methods. London: Sage Publications.

De Ruyter, K. 1996. Focus versus nominal group interviews: A comparative analysis. Marketing Intelligence \& Planning 14(6): $44-50$.

Doorn, N. 2017. Resilience indicators: Opportunities for including distributive justice concerns in disaster management. Journal of Risk Research 20(6): 711-731.

Eckstein, D. 2018. Global climate risk index 2018. https://german watch.org/sites/germanwatch.org/files/publication/20432.pdf. Accessed 16 Jan 2020.

Flick, U. 2014. An introduction to qualitative research, 5th edn. London: Sage Publications.

Frey, J.H., and A. Fontana. 1991. The group interview in social research. The Social Science Journal 28(2): 175-187.

Gaillard, J.C., and R. Jigyasu. 2016. Measurement and evidence: Whose resilience for whom? Resilience Development Initiative. https://www.preventionweb.net/files/50932_50932wpnov2016 gaillardjigyasu.pdf. Accessed 16 Jan 2020.

Grayson, R.B., B.L. Finlayson, C.J. Gippel, and B.T. Hart. 1996. The potential of field turbidity measurements for the computation of total phosphorus and suspended solids loads. Journal of Environmental Management 47(3): 257-267.

Gregorowski, R., A. Dorgan, and C. Hutchings. 2017. Resilience measurement-MEL Approaches in practice. Challenges and lessons in operationalizing resilience measurement frameworks-Experience and lessons from CoP stakeholders. Hove, England: ITAD Ltd.

Johnson, J.M., and T. Rowlands. 2012. The SAGE handbook of interview research: The complexity of the craft. Thousand Oaks: Sage Publications.

Konrad, C.P. 2003. Effects of urban development on floods. Fact sheet 076-03. Tacoma: United States Geological Survey.
Kotzee, I., and B. Reyers. 2016. Piloting a social-ecological index for measuring flood resilience: A composite index approach. Ecological Indicators 60: 45-53.

Kulig, J.C., D.S. Edge, I. Townshend, N. Lightfoot, and W. Reimer. 2013. Community resiliency: Emerging theoretical insights. Journal of Community Psychology 41(6): 758-775.

Kusumastuti, R.D., Viverita, Z.A. Husodo, L. Suardi, and D.N. Danarsari. 2014. Developing a resilience index towards natural disasters in Indonesia. International Journal of Disaster Risk Reduction 10(Part A): 327-340.

Kwok, A.H., E.E.H. Doyle, J. Becker, D. Johnston, and D. Paton. 2016. What is 'social resilience'? Perspectives of disaster researchers, emergency management practitioners, and policymakers in New Zealand. International Journal of Disaster Risk Reduction 19: 197-211.

Leximancer. 2017. Leximancer User Guide Release 4.5. https://www. coursehero.com/file/41515975/LeximancerManualpdf/. Accessed 15 Jul 2017.

Lindenmayer, D., J. Pierson, P. Barton, M. Beger, C. Branquinho, A. Calhoun, T. Caro, H. Greig, et al. 2015. A new framework for selecting environmental surrogates. Science of the Total Environment 538: 1029-1038.

Marshall, C., and G.B. Rossman. 2014. Designing qualitative research, 5th edn. London: Sage Publications.

Mayunga, J.S. 2007. Understanding and applying the concept of community disaster resilience: A capital-based approach. Summer Academy for Social Vulnerability and Resilience Building 1: Article 16.

Mellin, C., S. Delean, J. Caley, G. Edgar, M. Meekan, R. Pitcher, R. Przeslawski, A. Williams, and C. Bradshaw. 2011. Effectiveness of biological surrogates for predicting patterns of marine biodiversity: A global meta-analysis. PLoS One 6: Article e20141.

Miguntanna, N.S., P. Egodawatta, S. Kokot, and A. Goonetilleke. 2010. Determination of a set of surrogate parameters to assess urban stormwater quality. Science of the Total Environment 408(24): 6251-6259.

Patton, M.Q. 1990. Qualitative evaluation and research methods. London: Sage Publications.

Peacock, W.G., S.D. Brody, W.A. Seitz, W.J. Merrell, A. Vedlitz, S. Zahran, R.C. Harris, and R. Stickney. 2010. Advancing the resilience of coastal localities: Developing, implementing, and sustaining the use of coastal resilience indicators: A final report. Galveston: The Center for Texas Beaches and Shores (CTBS), Texas A\&M University at Galveston.

Penn-Edwards, S. 2010. Computer aided phenomenography: The role of Leximancer computer software in phenomenographic investigation. The Qualitative Report 15(2): 252-267.

Peters, K., A. Bahadur, T. Tanner, and L. Langston. 2016. 'Resilience'across the post-2015 frameworks: Towards coherence? London: Overseas Development Institute.

Qasim, S., M. Qasim, R.P. Shrestha, A.N. Khan, and K. Tun. 2016. Community resilience to flood hazards in Khyber Pukhthunkhwa province of Pakistan. International Journal of Disaster Risk Reduction 18: 100-106.

Rockström, J. 2003. Resilience building and water demand management for drought mitigation. Physics and Chemistry of the Earth, Parts A/B/C 28(20): 869-877.

Rodrigues, A.S.L., and T.M. Brooks. 2007. Shortcuts for biodiversity conservation planning: The effectiveness of surrogates. Annual Review of Ecology, Evolution, and Systematics 38: 713-737.

Saja, A.M.A., A. Goonetilleke, M. Teo, and A.M. Ziyath. 2019. A critical review of social resilience assessment frameworks in disaster management. International Journal of Disaster Risk Reduction 35: Article 101096. 
Saja, A.M.A., M. Teo, A. Goonetilleke, and A.M. Ziyath. 2018. An inclusive and adaptive framework for measuring social resilience to disasters. International Journal of Disaster Risk Reduction 28: 862-873.

Schoch-Spana, M., K. Gill, D. Hosangadi, C. Slemp, R. Burhans, J. Zeis, E.G. Carbone, and J. Links. 2019. The COPEWELL rubric: A self-assessment toolkit to strengthen community resilience to disasters. International Journal of Environmental Research and Public Health 16(13): Article 2372.

Sharifi, A. 2016. A critical review of selected tools for assessing community resilience. Ecological Indicators 69: 629-647.

Sotiriadou, P., J. Brouwers, and T.-A. Le. 2014. Choosing a qualitative data analysis tool: a comparison of NVivo and Leximancer. Annals of Leisure Research 17(2): 218-234.

UNISDR (United Nations International Strategy for Disaster Reduction). 2015. Sendai framework for disaster risk reduction 20152030. Geneva: UNISDR.
Yamamoto, L., D.A. Serraglio, and F.D.S. Cavedon-Capdeville. 2018. Human mobility in the context of climate change and disasters: A South American approach. International Journal of Climate Change Strategies and Management 10(1): 65-85.

Yin, R.K. 2013. Case study research: Design and methods. London: Sage Publications.

Ziyath, A.M., M. Teo, and A. Goonetilleke. 2013. Surrogate indicators for assessing community resilience. In Proceedings of the international conference on building resilience, 17-19 September 2013, Ahungalla, Sri Lanka. https://eprints.qut.edu. au/63404/1/Surrogate_indicators_for_assessing_community_resi lience.pdf. Accessed 10 Jan 2017.

Zubair, L., V. Ralapanawe, U. Tennakoon, Z. Yahiya, and R. Perera. 2006. Natural disaster risks in Sri Lanka: Mapping hazards and risk hotspots. In Natural disaster hotspots case studies, ed. M. Arnold, R.S. Chen, U. Deichmann, M. Dilley, A.L. LernerLam, R.E. Pullen, and Z. Trohanis, 109-136. Washington, DC: World Bank. 